the affected wounds or ulcers sometimes generate a subtle excreted matter, transmissible through the medium of the atmosphere, and capable, like small-pox, of exciting like affections in others. In proof of this, Brugmans mentions, that in 1798 , when hospital gangrene prevailed in one of the lower wards at Leyden, while the ward above was exempt, an opening for the purpose of ventilation was made in the partition ceiling that divided them, and in thirty hours afterwards three of the patients who lay next the opening were attacked by the disease. Professor Brugmans also ascertained, by analysis of the air in the wards, that the oxygen is diminished, the quantity of nitrogen and carbonic acid increased in proportion, while sulphuretter hydrogen could be detected as being present. From all of these facts the deduction follows-lst, that hospital gangrene, originating as a local disease in a wound or ulcer, can be propagated from one individual to another by transference, or inoculation, of a specific morbid poison, and that this inoculation is followed by constitutional fever of an inflammatory or typhoid type, according to the predisposed state of individuals; 2nd, that in over-crowded hospitals it is also transmissible through the medium of the atmosphere, and that the specific morbid poison excreted by the sore may follow as the result of constitutional causes, or specific fevers, and is then usually preceded by febrile symptoms of a continued bilious or remittent type.

Treatment. - In the treatment of hospital gangrene it will be necessary to weigh well all the circumstances of its probably local nature, or as the secondary result of primary specific fever or predisposed scorbutic constitution. The system of treatment must be either remedial or prophylactic. If the local symptoms are of a specific origin, and precede the constitutional ones, the local remedial measures necessary are applications of varions caustic substances, as undiluted nitric acid or caustic potass, which destroy the gangrenous slough, and prevent absorption of the septic matter into the constitution; vinegar has also been found usefal, and is one of the applications recommended by Paulus Agineta. Lemon-juice is the usual application in the French hospitals, and Mr. Nelaton employs the tincture of iodine with very great benefit. Or when the local disease seems to be rather the result of an excess of unhealthy inflammatory action in a part, the use of various soothing medi cated applications, such as finely-powdered charcoal and opium, chlorides of lime and soda, with the addition of opium, may be preferable. The latter, while they tend to reduce the excessive inflammatory action, materially arrest, by their chemical agency, the progress of putrefaction and constitutional contamination. Carrot or pumpkin poultices, mixed up with an oily solution of extract of hemlock, will be found to be a very soothing application in warm climates. If the constitutional symptoms be of an inflammatory kind, bloodletting may be requisite; and under all modifications of febrile attacks, saline eliminants of acetate and nitrate of potass, with nitrous ether and antimonial wine, are indispensable remedial means. Bleeding was found very efficacious by Dr. Boggie in the hospital gangrene that prevailed at Bilboa.

But while remedial measures are made use of, prophylactic measures should never be neglected, more particularly as the accumulation of septic matter in the atmosphere materially aggravates cases of the disease. Segregation of the patients, therefore, as far as circumstances may admit, should be carefully attended to. All cases of dysentery and typhoid fever should be also separated from cases of wounds and ulcers. But when segregation cannot be effectually carried out, the local use of charcoal to the sores-and the sanitary use of it as a disinfecting agent in the hospital wards, as recommended by Dr. Stenhouse, of St. Bartholomew's Hospital-must be assiduously applied. The extraordinary efficacy of charcoal as a deodorizing and disinfecting agent arises from its power of absorbing and oxidizing the bad air of over-crowded hospitals, and great credit is due to Dr. Stenhouse for the open, liberal spirit in which he has made public this important discovery.

Gutta Percha as an Eotrotic.-A solution of gutta percha in chloroform as an ectrotic in small-pox has been recently much recommended by $\mathrm{Mr}$. Wallace of Greenock. It seems to possess many advantages over collodion in preventing pitting in this disease. Collodion, as originally suggested by Dr. Ranking in THE LANCET, Mr. Wallace found veryeffectual in eleven cases; the application was, however, attended with pain, heat, and tension. These objections, he believes, are entirely obviated by the solution of gutta-percha, which the surgeon should be careful to paint on at the proper time-viz., immediately before complete maturation of the vesicles.
CONTRIBUTIONS TO

\section{THE PHILOSOPHY OF ZOOLOGY,}

WITA SPECIAL REERRENCA TO

THE NATURAL HISTORY OF MAN.

By ROBERT KNOX, M.D., F.R.S. EDIN.,

LECTURER ON ANATOMY, AND CORRESPONDING HFMBTR OF THE AEDDEMT OF MEDICINE OF ERANCE.

(Continued from vol. i. p. 627.)

"A magnificent temple is a laudable monument of national taste and religion; and the enthusiast who entered the dome of St. Sophia might be tempted to suppose that it was the residence, or even the workmanship, of the Deity. Yet how dull is the artince, how ensignifeant is the labour, if it be compared with the formation of the vilest insect that crawls upon the surface of the temple!"-Gibson. Docline and Fall.

\section{PART I.}

Introduction.-The philosophy of zoology can be based only on anatomy. Men, it is true, of vast genius had attempted, and with some success, to formule the laws regulating or presiding over the formation of animal bodies-the laws, in fact, regulating animal forms-by a bold synthesis, independent of all analysis. Such were the attempts of Aristotle, and of Newton. Buffon, availing himself, no doubt, to a certain extent of the labours of Daubenton and others, but more especially influenced by the comprehensive views which a deep and constant meditation of Nature taught him to adopt, made an effort in in the same direction, sufficiently powerful to attract the atten. tion of the Sorbonne and the Vatican. But so long as the philosophy these great men taught or proposed was without a basis in anatomical research, so long was it entitled only to the name of "hypothesis," unsupported by proof, by an appeal to intuitive evidence, or by any analysis of the material composing the essence of animal forms. Their views, then, were, by all inquirers into truth, regarded-and this, in the nature of things, could not well be otherwise-merely in the light of ingenious hypotheses, grouping together phenomena of which the greater part were avowedly mysterious and inexplicable.

So long as the laws of zoology were confined to, and embraced within, the doctrine of tinal causes, all philosophic minds were of course sensible that the philosophy of natural history, including, of course, zoology, could have no existence; for these doctrines placed it of necessity within that ultimate appeal, the First Cause, where all science ceases. The principle, or rather the phenomena, of life, so mysterious in their nature, so difficult to investigate, led no doubt to this view-to this ultimate appeal. It was in vain that gifted men, as Fontana and others, mathematicians and geometricians, pointed out that final causes were not causes, but effects; the doctrine maintained its ground, and does so still. The successive philosophies of the day, as they were called-the theological. namely, and the metaphysical-gave the doctrine sufficient support to overrule the natural philosopher, and to remove the phenomena of life, at least, from his field of inquiry. The reason was this: the field of inquiry included MAN, whose obvious connexion with the zoological world could not be mistaken, could not be denied. But man's history had been made the subject of elaborate works by historical and theologicohistorical writers; the animal and vegetable worlds had been described as subservient to him; whatever trenched on histories which, taken literally, are clearly and simply fabulous, gave alarm to powerful classes of men, deeply and intensely interested in obstructing all inquiry into truth, and Goëthe, Oken, and Geoffroy St. Hilaire would have shared the fate of Galileo, but for the accidentally altered circumstances of the European world. The French revolution had occurred-that mightiest of all human events. The artificial bonds of society were loosened. Men for the first time ventured to think. The mass of mankind rose incredibly in intellectual calibre for a shert space; it attempted to soar with science into the loftier, purer, and calmer atmosphere of the thinking world; its natural grossness brought it rapidly to the earth, where it now lies, a prey to every imposture, the struggle serving only to rivet each chain more securely than before. In the struggle, science escaped, not unobserved by the natural enemies of truth, repudiating alike the deceiver and the deceived. But great as was the genius of Goëthe, Oken, and Geoffroy, it would have proved unavailing to stem, even for an instant, the vast turbid current of folly and fable which passed even to later times for a history of this globe and its living inhabitants, but for the accidental appearance of one man, who unconsciously submitted to thinking men, not a hypothesis, not 
a theory, but a demonstration, irrefutable as those of Euclida demonstration of the fact, that all previous histories of the globe and its living inhabitants were simply fables. That man was George Cuvier, an anatomist. Palissy, it is true, had attempted this demonstration long before, but Palissy was a potter, not an anatomist. Buffon threw out a bold conjecture, and was instantly silenced by the Church of Rome. Cuvier's demonstration could not be so disposed of; it admitted of no refutation. The most subtle Jesuit, ever ready with a refutation even of Newton, quailed before the organic remains, attesting the existence of a former world, of a vast, if not of an infinite, antiquity.

It is worthy of remark, that Goëthe, Oken, Geoffroy, and Cuvier, however much they might differ on some points, agreed in this : that the basis of Philosophic Zoology was to be sought for in Anatomy. Goëthe saw this at once, even when a mere student; so did Oken, Cuvier, and Geoffroy. Comparative anatomy was the starting-point of all, but from this their course was different, divergent; Goëthe, by the transcendant force of his genius, was straightforward, cautious, and philosophical; Oken, theoretical, transcendental, and mythical; Geoffroy halted between the systems; Cuvier refusing the aid of embryology, preferred a perpetual recurrence to the miraculous interposition of supreme power-a frank confession that with him science and philosophy no longer guided his pen.

The discovery of the signification of the fossil remains (ossemens fossiles) led Cuvier to the conclusion, startling to the thinking mind, the philosophic,-namely, that there had been three or four successive creations of the animal and vegetable worlds. As he grew older, and his intellect stronger, he himself felt the antagonism existing in such a view to sound philosophy, and he committed to paper a direct contradiction to those who had ascribed such an opinion to him. But still later he returned somewhat to his original views-the riews of his early years. Goëthe, Oken, Geoffroy, never changed; as at first, so at last: one creation was their motto; unity of organization; unity of type; unity of plan, was their theory. Not so expressed it is true, and variously modified by each, but still based on one great idea. Unity of plan, unity of design, observable, traceable, demonstrable, in all forms which live, which have lived, or which may hereafter come into being.

I commenced these inquiries in 1810 , and since that moment have never lost sight of them. My first endeavour was to reduce the structure of the fore-foot of the horse to the corresponding parts in man; to compare and to discover the corresponding structures. I felt sure that they must have been formed on one plan; it was a deep conviction, arrived at by no reasoning-instinctive. As might be supposed, my success at that time was not great. The rudimentary fingers in the horse surprised me. Why rudimentary fingers? I said to myself. I fancied that all Nature's works were perfect. The theory of arrested development, at which Meckel and the German school caught so strongly, did not satisfy me, and never did, even when forced to teach it for want of a higher generalization. It is a doctrine I am now prepared to refute. The laws of deformation are as regular as the laws of formation. My next effort (1812) was to compare the organs of sight in man with the same in all other animals. Other structures followed, in all the natural families from man downwards. Satisfied, by a constant appeal to structure, that one great plan regulated all-that there could not be two or more planstwo or more creations - I had not yet seen any of the founders of the philosophy of zoology. This happened in 1821 . Acquainted with what I had done for comparative anatomy, no introduction was required to those whom I most desired to see-Cuvier, Geoffroy, and De Blainville: Goëthe and Oken were in Germany, and thus I never met the real founders of all philosophical anatomy. But my own dissections furnished me with data sufficient to prevent me coinciding with Geoffroy in his views of unity of the organization; whilst deep instinct, which never errs, told me that of the four creations maintained by Cuvier, three at least must be superfluous; to the geometrician $I$ leave the refutation of the one creation, intending not to meddle with that which seems beyond the reach of human thought. My object in this free inquiry is merely to ascertain, if possible, if there really be a philosophy in zoology-if its basis can be shown to rest on science-if zoological knowledge forms a part of science, or merely an amusing mode of contrasting the human with the non-human. As I view it, a history of zoology requires for its basis an inquiry into-

1. The laws of species and of natural families. Do species and natural families exist, or are these distinctions merely human contrivances resulting from imperfect observation? Is mankind composed of one great natural family, or of several ? and if of one, how many species, as permanent varieties are called, enter into its composition? Are species and natural families permanent and eternal, or is it merely a question of time? What part do embryonic forms play in Nature's grand scheme? Do they merely shadow forth a unity of plan, or do they, rather, pourtray the endless varieties of forms which animal matter may in time and space assume? In this view, the embryo is the most perfect of forms, embracing within it the possible of the past, the present, and the future. Individual adult forms simply show the development of that which can exist in unison with the existing order of things. The modern rhinoceros and elephant, tapir and sloth, are simply the developed forms of a natural family which once existed under other forms. They are not the mere hereditary descendants of the fossil world, as Geoffroy thought, but forms new in space and in time, but pre-existing in every embryo of their natural families. The embryo, then, is that which Nature perfects, that to which she looks for the continuation of varied life as it exists and has existed on the globe. The future is wrapt up in the same category. The embryo of any species of any natural family contains within it during its phases of development, all the forms or species which that natural family can assume or has assumed in past time. In the embryo and the young individual of any species of the natural family of the Salmonidæ, for example, you will find the characteristics of the adult of all the species. The same, I believe, holds in man; so that, were all the existing species of any family to be accident ally destroyed, saving one, in the embryos and young of that one will be found the elements of all the species ready to reappear to repeople the waters and the earth, the forms they are to assume being dependent on, therefore determined by, the existing order of things. With another order will arise a new series of species, also foreseen and provided for in the existing world. There can be no such thing as arrested development, nor a gradual development of all forms towards perfection. Nature's works are perfect, first and last; and the hypothesis which supports the idea of a development tending to perfection is simply another expression of the doctrine of a final cause. The so-called "arrest of development" in the individual is a doctrine admitting of the easiest refutation. Development can only mean development, and nothing else. This may be either individually and specifically progressive, or zoologically retrogressive-i. e., back towards other forms; but always development, notwithstanding-a retrogressive development. The laws of deformation are as constant and regular as the laws of formation, which we call perfect, as belonging to the existing world; this is all. But to enter on this inquiry, a preliminary investigation is necessary. Zoological forms either obey general laws or they do not; they have been formed on one great plan, or on several plans. If the latter be maintained, it ought to be shown in what the plans differ; if the former, the scheme or plan ought to be explained and its existence demonstrated, if possible. If no such plan exist, there exist no laws, which, indeed, in that case, are not required, the scientific basis on which zoology rests being removed, it would cease to be a science. Whether life was coeval with the globe or not, is a question not essential to the present inquiry. At its appear. ance it of necessity obeyed those laws which philosophy is called on to investigate. In their essence these laws can never alter: this is what science teaches. No fossil remains have ever been discovered contradictory of the theory that one great scheme or plan has at all times existed. In a strictly philosophical sense, there could be only one creacion; but the real difficulty is to determine what were the zoological forms of that creation. Were they species--that is, races, or did the individuals represent natural families embracing many species? However this may be, one thing is certain: many races or species have ceased to be, whilst others, which were not then, now live. These are new species merely as regards man, for, in point of fact, distinct species exist not in Nature. They are not included in the great scheme which fills up all voids, all differences, all distinctions. The gap-absence of the link connecting one species, as we call them, with another-man assumes as a positive fact in Nature-an intentional part of her plan; but it is not so, as science will soon demonstrate. Some persons have troubled themselves with a refutation of the hypothesis of the convertibility of one species into another. It is wholly a labour of supererogation, for no strictly scientific man ever entertained such an hypothesis in the sense assumed by them.

My first step, then, is to establish the doctrine of one great plan or type for all animals; a unity of plan - that is, a typea type " which exists everywhere, but is nowhere to be found." A material archetype is the invention of a mind sunk in materiality and in error. No such can exist, since a part can never represent a whole. 
A type being proved to exist, there arises next the question of varieties in zoological forms, embraced within the type. The application of these two inquiries includes all natural history as a science; if applicable to one they are applicable to all forms, man included. The law called " the arrest of development hypothesis" explains nothing, and involves increasing contradictions in terms. For many years I have preferred using the terms " persistence of embryonic forms," although this phrase also is open to manifest objections. The persistence of the webbing of the human fingers to the adult state has been described as " an arrest of development." I should have thought it rather " a retrogressive development" towards another form. Is the presence of a third and a fourth head of the biceps muscle in man "an arrest of development"? Is the presence of a supra-condyloid process in the humerus and the passage of the humeral artery and median nerve through the passage thus formed an arrested development? Is it even a persistence of embryonic forms? These doctrines are nearly the reverse of those I hold. In the embryo I see a perfect, not an imperfect being; its developments, transmutations, metamorphoses, follow certain laws; they equally tend to perfection of the individual species as a species, of the individual as an individual. But their developments must not be called imperfect, inferior, or arrested developments, merely because they happen to tend towards, and to produce, inferior forms of organization, as we esteem them. The brain of the human embryo, like all its other structures, no matter to what species it belongs, embraces every possible form which man assumes, or has assumed, on the earth. The white races are not the more fully developed, and the negro the more imperfectly developed, species of one common natural family. The development of each is perfect in its way-equally so. The negro brain is not an imperfectly developed white brain, but a specifically formed brain, developed according to its own specific laws. When in the white races we find developments resembling the negro, and vice vers $\hat{\alpha}$, these are not arrested developments, but retrogressive, if you will, yet perfect in their kind. To instance a class of animals, supposed, erroneously, however, to be less complex in their organization than man, lower in the scale, less perfect-the class Fishes; and selecting amongst these, as, perhaps, most familiar to my readers, the natural family Salmonida,--if in this natural family, divisible into three sub-families, I find the young of any of the species of any of the sub-families strictly to resemble each other, and yet the grown individual of any of the species materially to differ from any other, it by no means follows that the one is a species less developed than another. In the embryo-in the young-all are alike; each offers in itself the specific characters found in all. The embryo salmo, for example, is of no species; it is of all. Take from it one set of characters, it becomes the salmo; deduct another, you have the forelle; subtract a third, you have the trout. In every embryo, of every species, we hare the possible of all forms appertaining to that natural family at least. The destruction, then, by geological or other phenomena, of all the species, saving one, of any natural family, would not necessarily extinguish that family, since, in the embryo of the remaining species, there exist the elements for the reappearance, perhaps under modified forms, of all the extinct species. As of fishes, so of man: one natural family-one embryonic form, equal to the production of all species in accordance with the essential conditions of existence in time and space.

\section{ON THE \\ PROCESS OF THE ELIMINATION OF MORBID POISONS.}

BY JOHN GEO. FRENCH, Esq., F.R.C.S., SURGEON TO THE INYIRMARY OF ST. JAMIES'S, WESTMINSTEER.

THe term elimination is of modern date. It is not contained in Johnson's Dictionary; it is derived from elimine (from the threshold, ) and is sufficiently expressive. It is used by Mason Good as applied to the exanthematous eruptions which he says are "eliminated upon the surface as the best and most salutary outlet."

The process of the elimination of morbid poisons from the system is not only a subject but little understood, but very serious errors are generally entertained upon it. It may be remarked that all knowledge in the medical art has ever been but slowly acquired. It is but a short time since that the reparation of wounds was ascribed to inflammatory action, although it is now perfectly understood that inflammation is but a dis. turbing cause too apt to interfere with, and delay the process of reparation; and in point of fact the treatment of those injuries consists in avoiding the causes of inflammation, and placing the injured parts under such circumstances as the surgeon knows, from study of the natural mode of reparation, to be favourable to the process of cure. Any improvement in the medical art has always been evinced by greater simplicity of treatment, arising from more accurate knowledge of principles.

Now it may be stated as a general rule, and a most valuable maxim, that whenever Nature undertakes to perform a specitic process of elimination, the greatest possible care should be taken by the physician that no interference on his part should meddle with its accomplishment. "A meddlesome midwifery is a bad midwifery" was the frequent exclamation of an able teacher of his art; that a meddlesome physician is a bad physician is equally certain. In fact, the difference between one physician and another consists in the success with which each interprets Nature. Let us illustrate this position by reference to the exanthemata. In small-pox the treatment is founded on the theory that the skin secretes or separates the poison from the body, and it is on the knowledge of what this process is, as revealed by direct clinical observation alone, that the physician is of service in conducting a case in the path of recovery. It is a matter of history, that all attempts on his part at elimination have ever been attended with but one resultnamely, that of aggravating the disease; while successful treatment depends wholly on the power of the constitution in eliminating, and on the resources of the practitioner in removing all obstructions to the completion of this process; and, when this is accomplished, to sustain the patient during the extensive reparation which the skin has to undergo from the ravages of the disease upon it.* Again, in scarlet ferer the process of elimination is left to the natural efforts, whether this be by the skin or kidneys, the treatment, as far as this is concerned, being still to take care that these structures are placed under farourable circumstances for the performance of their functions. So, in cholera, if the alimentary canal be really performing the part of elimination, then the same rule applies - namely, that every obstruction to the performance of this function should be removed. To act consistently, we should totally abstain from giving aliment of any kind; it is unreasonable to expect that the alimentary canal can be assimilating and eliminating at the same time. We give ice on the same principle that we use cold ablution to the skin in certain states of fever-namely, to moderate excitement and relieve congestion, and thus remove obstruction to the perform. ance of the necessary elimination. It is not from any vague notions of either astringency or supposed power of quieting the stomach that ice is useful ; it is suggested by the direct elinical observation of Nature, and its use is analogous to that of easy posture and comfortable applications in the treatment of wounds. We give water to quench the thirst, refresh the patient, and restore the fluidity of the blood, as soon as the system is capable of receiving it. It is a great mistake to suppose that the study of the process of elimination, regarded as a natural function, can lead to any other view respecting eliminating medicines than to wholly condemn them as the result of experience and reason.

$\mathrm{I}$ am induced to bring this subject before the profession, in consequence of the publication of Dr. George Jolnson's views on the use of castor oil in the treatment of cholera, and I deem it necessary to show the fallacies of the reasoning by which it is recommended, and of the data by which it is supported.

Dr. Jolnson refers to the blood as the recipient of the poison, and says (page 257), "It would, perhaps, be difficult to prove that any purgative medicine has the power of directly assisting the escape of the cholera poison, or the products of that poison, from the blood into the intestine; but it cannot be doubted that, by removing the contents of the bowel, and so obviating the impediment to the secretory process which would result from their accumulation, an indirect aid is given to the elimination of the poison from the blood. The speedily fatal consequences of over-distention of the bowel, when the attempts to remove that condition have been unsuccessful, may be seen in Case 31 (p. 52.)"

Now, the theory of purgatives which act on the blood is this:

* About fifteen years since the late Dr. Gregory met a most intelligent friend of mine in consultation on a case of small-pox. The consultation was at a very early period of the disease, and on an early day in the week. "Well," said the doctor, "the ease is but slightly confuent, there is but intle fever, and no it riobt to apprise you that our patient will die on Friday"-an event verified by the result, in spite of the most unwearied efforts to avert it Dr. Gregory predicted this from his intimate knowledge of the natural history of the dis ease. He recognised in the appearanee of the eruption a want of vital power in the patient which would fail to accomplish the elimination of the poison. 